\title{
Characterization of Copper(II) complexes of Schiff base derived from benzil-2,4-dinitrophenylhydrazone with anilines using $x$-ray diffraction and Extended X-ray absorption Fine Structure (XRD and EXAFS)
}

\author{
Sushma Patidar $^{1 *}$, A. Mishra ${ }^{2}$, K.S. Sura ${ }^{3}$, S. Mohammad $^{4}$ \\ ${ }^{1}$ School of Physics, DAVV, Indore (M.P), India \\ ${ }_{3}^{2}$ School of Physics, DAVV, Indore (M.P), India \\ ${ }^{3}$ School of Physics, DAVV, Indore (M.P), India \\ ${ }^{4}$ School of Physics, DAVV, Indore (M.P), India \\ *Corresponding Author: sn2704@gmail.com, Tel.: +91-93014-47276
}

Available online at: www.isroset.org

Received: 20/Mar/2018, Revised: 31/Mar/2018, Accepted: 21/Apr/2018, Online: 30/Apr/ 2018

\begin{abstract}
Schiff base chelates of $\mathrm{Cu}$ (II) derived from benzil-2,4-dinitro-phenylhydrazone with different anilines have been synthesized. These complexes have been prepared by chemical root method. The XRD and EXAFS characterization of the prepared complexes have been carried out. From XRD particle size and lattice parameter have been estimated and the XRD analysis revealed the crystalline nature of all complexes. These complexes have also been characterized by $\mathrm{Cu} \mathrm{K}$-Edge EXAFS measurements using the dispersive beam line at $2.5 \mathrm{GeV}$ Indus-2 synchrotron radiation source at RRCAT (Raja Ramanna Centre for Advance Technology), Indore, India. The measured EXAFS data have been analyzed to compute average metal-ligand bond length using Levy's, LSS, Lytle's, Fourier transform methods, with the help of computer software ATHENA.
\end{abstract}

Keywords-XRD, EXAFS, Bond-length, Benzil-2,4-dinitrophenylhydrazone.

\section{INTRODUCTION}

Schiff bases have played an important role in the development of coordination chemistry. They are an important class of ligands and have broad applications in different fields. Interaction of these ligands with metal ions provides the complexes of various geometries which are most likely biologically active. From the existing literature, it appears that benzil monophenylhyrazone and its related compounds have been extensively used as biologically active complexing agents and analytical re-agents.[1,2]. Keeping the above facts in mind and in continuation of our research work on transition metal (II) complexes with Schiff bases, in the present paper we report the synthesis and characterization of $\mathrm{Cu}$ (II) complexes of Schiff base derived from the condensation of benzil-2,4-dinitrophenylhydrazone with different anilines[3].

XRD technique is used to show the details about the particle size, lattice parameter, and to know the crystal structure of these complexes. EXAFS spectroscopy has been a valuable tool to provide local structure.and gives the information about the chemical environment. The bond lengths has been calculated by Levy's , Lytle's and Lytle, Sayers and Stern's[LSS]methods.[4]

\section{MethodologY}

1.Synthesis of Schiff base Ligand L1/L2/L3:

The Schiff base was obtained by the condensation of benzil, 2,4-dinitrophenylhydrazine and aniline/o-Toludine/mToludine (1 :1: 1 molar ratio), dissolved in ethanol. The resulting reaction mixture was refluxed for $1 \mathrm{~h}$. The solid precipitate of Schiff base obtained was filtered, washed and then left in the air to dry.

\section{Synthesis of complexes:}

The solution of Schiff base L1/L2/L3 (10 mM) with ehanol was mixed with $\mathrm{Cu}$ (II) chloride $(5 \mathrm{mM})$ in ethanol $(20 \mathrm{ml})$ solution keeping ligand-metal ratio $2: 1$ followed by few drops of acetic acid $(\mathrm{pH}=6)$. The mixture was then refluxed for $1 \mathrm{~h}$. The soild precipitate of complexes has been filterd, washed and dried in air.

The above mentioned complexes have been characterized by $\mathrm{X}$-ray diffraction(XRD) and Extended X-ray Absorption Fine Structure(EXAFS).XRD pattern have been recorded by Bruker D8 advance diffractometer at UGC-DAE CSR, Indore. The X-ray absorption fine structure spectra at the Kedge of Copper of these complexes have been recorded at BL-8 Dispersive EXAFS beamline at the 2.5-GeV INDUS-2 
Synchrotron Radiation Source at Raja Ramanna Centre for Advanced Technology (RRCAT), Indore, India .The experimental data have been analyzed using software Origin 8.0 and Athena 0.8.056. [5].

\section{RESULTS AND DISCUSSION}

The analysis of crystal structure and lattice parameter have been carried out by Bragg's law, $2 \mathrm{~d} \sin \theta=\mathrm{n} \lambda$ and particle size is calculated by Debye Scherer's formula, $t=0.9 \lambda / \beta \cos \theta$, where $\lambda$ is the wavelength of $\mathrm{X}$-ray, $\theta$ is the glancing angle and $\beta$ is the FWHM ( Full width at half maxima).The XRD pattern of the reported complexes is presented in figure 1 . The particle size and lattice parameter are given in Table 1. The X-ray analysis shows the complexes are crystalline in nature.

The normalized X-ray absorption spectra of $\mathrm{Cu}$-complexes are reveled in figure 2 (a). The EXAFS spectra transformed into $\mathrm{k}$-space have been shown in figure 2(b). To convert into R-space a Fourier transform is applied to these data and it is shown in figure 2(c). The bond lengths of these $\mathrm{Cu}$-complexes have been calculated by different methods. The phase-uncorrected bond length has been calculated by the Fourier transform technique. Before Fourier transform technique was formulated, the bond lengths were extracted from the EXAFS data by the three methods:

\section{(A) Levy's method}

According to this method the bond length $\mathrm{R}_{1}$ is given by-

$$
\mathrm{R}_{1}=\left(\frac{151}{\Delta \mathrm{E}}\right)^{1 / 2} \AA
$$

where $\Delta \mathrm{E}$ is energy difference between the successive EXAFS maxima and minima.

\section{(B) Lytle's method}

\section{In this method, the bond length is calculated by}

$$
\mathrm{R}_{\mathrm{s}}(\AA)=\{37.60 / \mathrm{M}\}^{\frac{1}{2}}
$$

Where M is the slope of E versus Q plot.

\section{(C) L.S.S. method (Lytle, Sayers and Stern Method)}

The LSS method is a graphical method and according to this method, the simplified equation for wave vector is given by $\mathrm{k}$ $=(0.263 \mathrm{E})^{1 / 2}$ where, $\mathrm{E}$ is energy position of different maxima and minima in fine structure curve measured from point in $\mathrm{K}$ absorption edge. And the bond length is given by

$\mathrm{R}-\alpha=(\pi / 2) *$ slope of $\mathrm{n}$ vs $\mathrm{k}$ plot.

Where $\mathrm{n}$ is order of maxima and minima in $\mathrm{k}$ vs $\chi(\mathrm{k})$ plot and $\mathrm{k}$ is wave no.

\section{Fourier Transform Method:}

From the Fourier transform of $\chi(\mathrm{k})$ verses $\mathrm{k}$ spectra shown in figure2(b) phase uncorrected bond lengths has been determined.

\section{Conclusion}

From the analysis of X-ray diffraction pattern, information regarding particle size and lattice parameter has been obtained and octahedral geometry of these complexes has been concluded. From the positions of the EXAFS maxima and minima, the bond lengths of the $\mathrm{Cu}$-complexes has been determined by three different methods viz. Levy's, Lytle's and LSS methods and from the Fourier transforms of the EXAFS spectra, the bond length (phase uucorrected) has been determined.

Figures and Tables

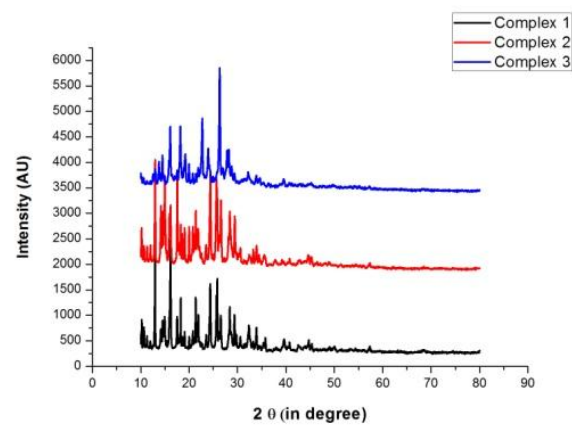

Figure 1 XRD of Cu Complexes

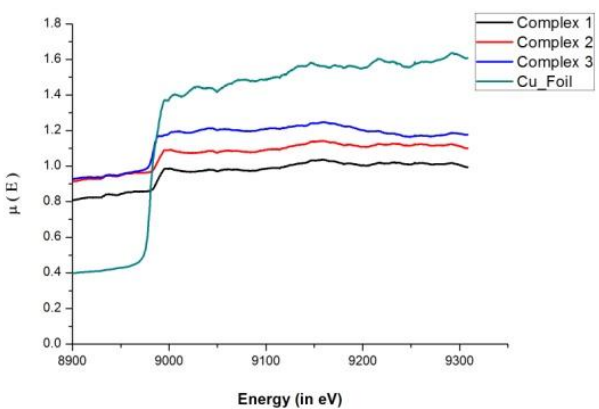

Fig 2(a)

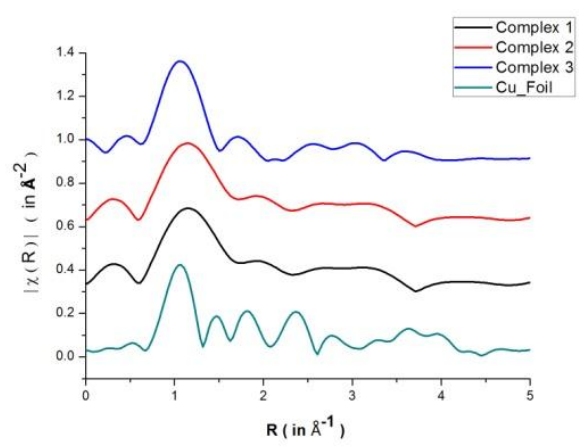

Fig 2(b) 


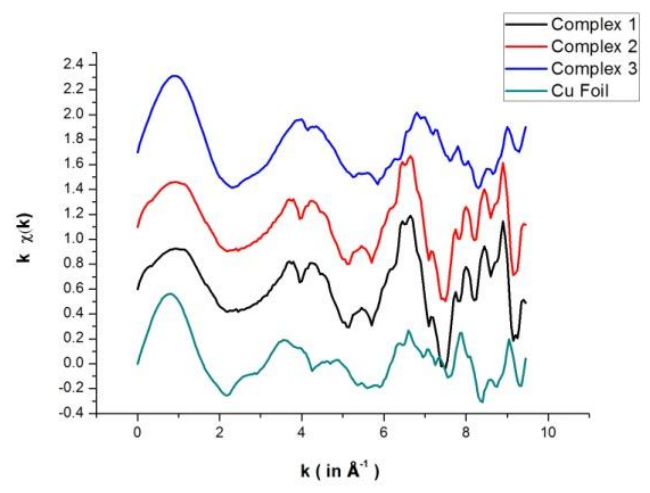

Fig 2(c)

Figure 2. For complexes 1-3 a) EXAFS spectra at the $\mathrm{Cu}$-K-edge (b) $\chi(\mathrm{k})$ vs. k spectra, and (c) Fourier transformation of $\chi(\mathrm{k})$ vs. k spectra.

Table 1.(Partical Size and Lattice parameter by XRD)

\begin{tabular}{|c|c|c|c|}
\hline S.No. & Name of Complexes & $\begin{array}{l}\text { Particle } \\
\text { size(nm) }\end{array}$ & $\begin{array}{c}\text { Lattice } \\
\text { parameter (Å) }\end{array}$ \\
\hline $\mathbf{1}$ & Complex 1 & 49.77 & 4.08 \\
\hline & & & \\
\hline $\mathbf{2}$ & Complex 2 & 40.47 & 4.62 \\
\hline & & & \\
\hline 3 & Complex 3 & 39.55 & 5.08 \\
\hline
\end{tabular}

Table 2.(Values of bond lengths calculated by Levy's, Lytle, LSS and Fourier transform methods)

\begin{tabular}{|c|l|l|l|l|l|}
\hline S.No. & $\begin{array}{l}\text { Name of } \\
\text { Complexes }\end{array}$ & \multicolumn{2}{|c|}{$\begin{array}{l}\text { Phase corrected } \\
\end{array}$} & & \multicolumn{2}{c|}{\begin{tabular}{l} 
Phase uncorrected \\
\hline
\end{tabular}} & $\begin{array}{l}\text { Levy's } \\
\text { method } \\
\mathrm{R}_{1}\end{array}$ & $\begin{array}{l}\text { Lytle } \\
\text { method } \\
\mathrm{R}_{\mathrm{s}}\end{array}$ & $\begin{array}{l}\text { L.S.S.method } \\
\text { R1- } \alpha 1\end{array}$ & $\begin{array}{l}\text { F.T } \\
\text { Method } \\
\mathrm{R}\end{array}$ \\
\hline 1 & Complex 1 & 1.82 & 1.49 & 1.9 & 1.15 \\
\hline 2 & Complex 2 & 1.68 & 1.51 & 1.88 & 1.13 \\
\hline 3 & Complex 3 & 1.47 & 1.47 & 1.97 & 1.2 \\
\hline
\end{tabular}

\section{ACKNOWLEDGMENT}

I would like to express my sincere gratitude to Dr Pratibha Sharma, School of Chemical Sciences, DAVV for providing the valuable guidance during synthesis of complexes, Dr. S N Jha, RRCAT, Indore, India for EXAFS measurents and Dr M. Gupta for providing facilities at DAE-CSR Indore.

\section{REFERENCES}

[1]. A K Singh and U N Sharma Asian J. Chem. 14, 1221, 2002.

[2]. N Raman ,A Kulandaisamy , C Thangaraja and K Jeyasubramanian Transition Met. Chem. 28, 29, 2003.

[3]. N Raman, S Ravichandran and C Thangaraja," Copper(II), cobalt(II), nickel(II) and zinc(II) complexes of Schiff base derived from benzil-2,4-dinitrophenylhydrazone with aniline", J. Chem. Sci., Vol. 116, No. 4, , pp. 215-219, July 2004

[4]. A Mishra, N Parsai, N Dagonkar, "Theoretical analysis of EXAFS data of Cu(II) complexes of isoxezol series", Indian Journal of Pure \& Applied Physics, Vol 47, pp 337-339,2009.

[5]. A Gaur, A Johari, B D Shrivastava, D Gaur, S N Jha, D Bhattacharyya, A Poswal and S K Deb, Sadhana 36, 339, 2011

[6]. J Bhale, P.Sharma, A Mishra, N Parsai, "Determination of bond length from EXAFS spectra of some copper(II) mixed ligand complexes", International Journal of Scientific Research in Physics and Applied Sciences, Vol 3,No.3,pp1-3,2015.

[7]. B. D. Cullity, "Elements of X-ray diffraction", Addison-Wesley publishing company,Inc.. , 1956.

[8]. Introduction to XAFS: A Practicla guide to X-Ray Absorption Fine Structure Spectroscopy by Grant Bunker Cambridge University Press 978-0-521-76775-0,2010 